\title{
Quantum phenomena in transport measurements of topological insulator nanostructures
}

\author{
(Review Article) \\ Lei Fang ${ }^{1,2}$ and Wai-Kwong Kwok ${ }^{1}$ \\ ${ }^{1}$ Materials Science Division, Argonne National Laboratory, IL, 60439, US \\ ${ }^{2}$ Department of Chemistry, Northwestern University, IL 60208, US \\ E-mail: lfang@anl.gov
}

Received October 29, 2013

\begin{abstract}
We review the recent experimental advances on quantum phenomena in transport measurements of topological insulators with emphasis on quantum oscillation, weak antilocalization and Aharonov-Bohm effect and Altshuler-Aronov-Spivak effect. Following a brief introduction on the topic, we discuss the identification of the topological surface state based on quantum phenomena. Research prospect of topological insulators is described at the end of this article.
\end{abstract}

PACS: 73.23.-b Electronic transport in mesoscopic systems;

71.70.Di Landau levels;

73.25.+i Surface conductivity and carrier phenomena.

Keywords: topological insulator, quantum oscillation, nanoribbon.

Contents

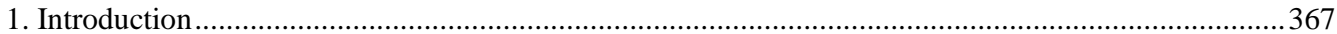

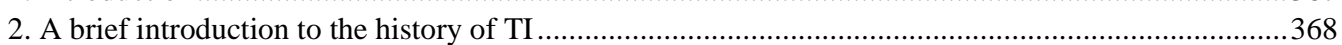

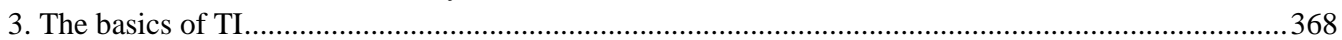

4. Quantum phenomena in the transport measurements on TI nanostructures …....................................369

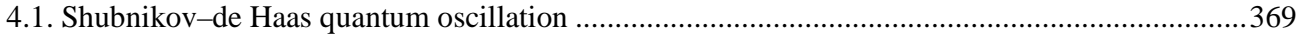

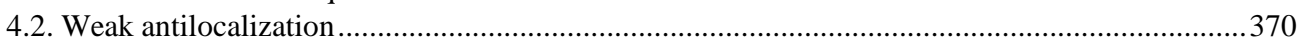

4.3. Aharonov-Bohm effect and Altshuler-Aronov-Spivak effect.....................................................371

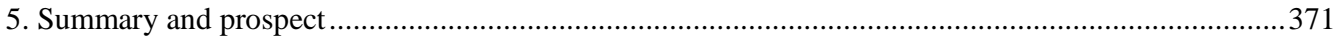

\section{Introduction}

In modern condensed matter physics, the notion of spontaneous symmetry breaking accompanied with the formation of an order parameter is generally applied to phase transitions in various macroscopic systems [1]. For example, translation symmetry is broken in the ground state of a magnet due to the alignment of the magnetic moments. In a conventional superconductor, the offdiagonal long-range order breaks global gauge symmetry [2]. A notable exception to this universal rule is quantum matter that is characterized by topological invariance. Such a topological phase cannot be described by any local order parameter. Prototypical materials that manifest this behavior are the recently discovered topological insulators (TIs) [3-7]. These materials are bulk insulators, that possess conducting edges or surfaces. Due to their special topological invariance, abundant novel phenomena have been ob- served in the TIs, such as the back-scattering immunity, Dirac cone-like electronic structure, and the helical spin structure. These remarkable properties could significantly benefit novel applications in fields such as spintronics [8,9] and quantum computing [10-13].

Here we briefly overview the quantum phenomena in transport measurements of TI nanostructures. In addition, a historical perspective of TIs and a summary of the basic properties of TIs are also presented. This paper is organized as follows. Section 2 is a brief introduction to the history of TI. Section 3 introduces the basic characteristics of TI. In Sec. 4, quantum phenomena, such as quantum oscillations and phase interference in TIs are discussed. The last section presents a prospect of TIs. This article is not intended as a comprehensive review. Readers who have interest in the theory and the characterization of TIs can refer to reviews [14-19]. A review of progress regarding the variety of TI materials can be found in [20,21]. 


\section{A brief introduction to the history of TI}

In modern condensed matter physics, theory plays an important role in predicting new phenomena. The research of TIs demonstrates the formidable power of theory and computation techniques to predict new materials and phenomena. Both the two-dimensional (2D) and the threedimensional (3D) TIs were first theoretically predicted, and later verified by experiments [3-7,22-32]. More recently, the anomalous quantum Hall effect was predicted in TIs doped with magnetic ions [33]. This prediction, again, was verified by experiments [34,35]. An examination of the "theoretical cradle" of TI is thus useful for both experiments and materials research.

In 1980, the integer quantum Hall effect (IQHE) was discovered in the two-dimensional electron gas (2DEG) in semiconductor heterostructures at low temperatures and high magnetic fields [36]. It was soon realized that the IQHE can be described as a topological phase [37] with a chiral edge state [38]. In such a state, electrons can flow without dissipation [38]. Although desirable for device applications, IQHE usually requires high field to quantize the electrons. This factor motivated theorists to explore the anomalous Hall effect without magnetic fields. Murakami, Nagaosa, and Zhang took spin-orbit coupling into consideration and created the concept of a spin Hall insulator [39]. In semiconductors HgTe and PbTe, the heavy-hole and light-hole bands have opposite signs for the mass and a finite spin-Hall effect was predicted to appear under the condition that only the heavy-hole bands are occupied at zero doping. In 2005, Kane and Mele improved the concept and performed calculations for the quantum spin Hall insulator (QSHI) [3,4]. In the Kane-Mele model, the effects of the spin-up and spin-down electrons were considered equivalent to that of the magnetic fields. Applying two copies of the Haldane model which was originally created to explain the IQHE in a magnetic field [40], Kane and Mele found that QSHI has conductive edge states. The electrons of the edge states are immune to impurity scattering as long as the time reversal symmetry (TRS) of the edge states is preserved. In 2006, Bernevig and Zhang proposed the quantum spin Hall effect (QSHE) in semiconductor quantum wells [5,6]. The strong spin-orbit coupling (SOC) in the quantum wells inverts two electronic bands to cross the band gap and form a Dirac-cone-like energy spectrum. Their prediction was experimentally confirmed a year later by Molenkamp's group in quantum well CdTeHgTe-CdTe [7]. The QSHE is not limited to 2D systems. In 2007, Moore and Balents generalized the Kane-Mele model in an arbitrary multiband band structure in two or three spatial dimensions [22]. In the same year, $\mathrm{Fu}$ and Kane predicted that the alloy $\mathrm{Bi}_{1-x} \mathrm{Sb}_{x}$ is a 3D TI [23,24]. In 2008, Hasan's group at Princeton University successfully observed the Dirac-cone-like electronic structure in $\mathrm{Bi}_{1-x} \mathrm{Sb}_{x}$ by using angle resolved photoemission spectroscopy (ARPES) [26]. Another confirmation of the topological state surface of $\mathrm{Bi}_{1-x} \mathrm{Sb}_{x}$ came from angle dependent quantum oscillation measurements [30]. However, the electronic structure of $\mathrm{Bi}_{1-x} \mathrm{Sb}_{x}$ is extremely complicated. This prompted an intense search for new 3D TIs. An important progress on 3D TI came in 2009. One group at the Institute of Physics at Beijing proposed that the narrow band semiconductor $\mathrm{Bi}_{2} \mathrm{Se}_{3}$ and $\mathrm{Bi}_{2} \mathrm{Te}_{3}$ are $3 \mathrm{D}$ TIs that have a single Dirac cone [25]. The 2D surface channel was soon confirmed by angle dependent photoemission spectroscopy and quantum oscillation measurements [26-29,31,32].

\section{The basics of TI}

The strong SOC generates a Dirac-cone-like energy spectrum in the band gap of a TI, as shown in Fig. 1(a). The linear dispersion leads to two predominant characteristics of the Dirac electrons: zero mass and the high mobility. In reciprocal space, Dirac cones of TI are only located at the Kramer points, for instance the $\Gamma$ point or the $k$-point of a Brillouin zone, where $k=\pi=-k$. This feature is determined by the Kramer theorem: in a TRS system, if $\mathcal{T}^{2}=-1$, all the energy levels must be doubly degenerate [41], where the $\mathcal{T}$ is the TRS operator. Figure. 1(b) (a)

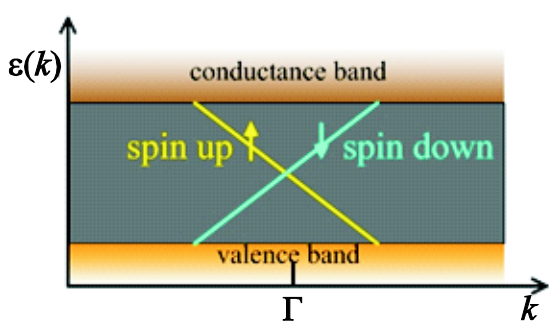

(b)

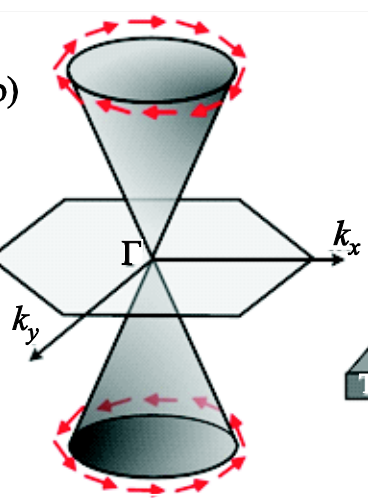

(c)

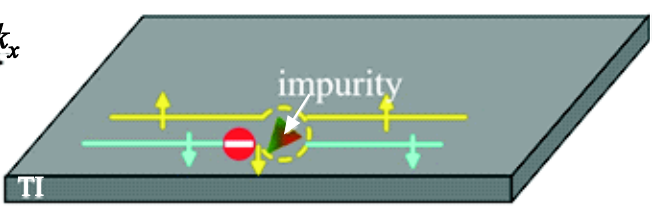

Fig. 1. (Color online) Dirac-cone-like energy spectrum in the band gap of a TI (a). Schematic picture of a Dirac cone of TI in 3D reciprocal space. The red arrows represent the spins of the Dirac electrons. The spin direction is perpendicular to the electron's momentum and forms a unique helical spin structure (b). The immunity to backscattering in a TI. The triangle denotes a static impurity (c). 
Table 1. The comparison between IQHE and SQHE

\begin{tabular}{|c|c|c|c|c|c|c|c|}
\hline & $\begin{array}{c}\text { Topological } \\
\text { invariance }\end{array}$ & TRS & SOC & Edge state & Impurity scattering & Transport & Dimensionality \\
\hline IQHE & Yes & Broken & Unrelated & $\begin{array}{c}\text { Chiral or skipping } \\
\text { orbit }\end{array}$ & No & $\begin{array}{l}\text { Quantized } \\
\text { transvers con- } \\
\text { ductivity }\end{array}$ & $\begin{array}{l}\text { Only even } \\
2 \mathrm{D} \text { or } 4 \mathrm{D}\end{array}$ \\
\hline QSHE & Yes & Preserved & $\begin{array}{l}\text { Strongly } \\
\text { related }\end{array}$ & $\begin{array}{c}\text { Helical } \\
\text { spin structure }\end{array}$ & $\begin{array}{c}\text { Absence of } \\
\text { backscattering }\end{array}$ & $\begin{array}{l}\text { Longitudinal } \\
\text { conductivity in } \\
\text { units of } e^{2} / h\end{array}$ & 2D and 3D \\
\hline
\end{tabular}

schematically shows a single Dirac cone of TI in 3D reciprocal space. The red arrows represent the spins of the Dirac electrons. Measurement using spin-polarized ARPES found that the spins are locked perpendicular to the electron momentum and form a so-called helical spin structure $[27,42]$. The unique helical spin structure gives rise to a $\pi$ Berry's phase in the Dirac electrons.

Another fingerprint of the TIs is the absence of backscattering. As depicted in Fig. 1(c), the spin-up electrons and spin-down electrons counter-propagate due to time-reversal invariance (TRI). The triangle denotes a static impurity. If backscattering occurs, electron with either the spin-down or spin-up is scattered back with a flipped spin orientation. However, due to TRS, a state with two identical spin quanta in a single quantum channel is not allowed. Thus the backscattering process is prohibited. The immunity to backscattering in TIs has been observed in scanning tunneling spectroscopy experiments [43-45]. However, transport measurements found that the mobility of the Dirac electrons can be largely affected by the surface quality $[29,46]$, indicating that the complete mitigation of backscattering may not be achieved. This contradiction between experiment and theoretical prediction has not been fully addressed and more efforts are needed to clarify this point. A comparison between IQHE and QSHE is summarized in Table 1.

\section{Quantum phenomena in the transport measurements on TI nanostructures}

\subsection{Shubnikov-de Haas quantum oscillation}

The linear dispersion $\varepsilon(k)$ in TIs gives rise to zero-mass and highly mobile Dirac electrons. Hence a moderately high magnetic field is adequate to quantize the Dirac electrons into Landau levels. This enabled the observation Shubnikov-de Haas quantum oscillations in transport measurements of TIs [28,29,47-53]. To date, most experimental studies of TIs are based on $\mathrm{Bi}_{2} \mathrm{Se}_{3}$ and $\mathrm{Bi}_{2} \mathrm{Te}_{3}$ because of their relatively simple electronic structures and the availability of large crystals. However, there are large amounts of dislocations in naturally grown crystals of these materials [47]. These dislocations donate electrons and elevate the Fermi level, $E_{F}$, to the bottom of the conductance band. The coexistence of bulk and surface carriers leads to many ambiguities in the transport data. For example, in naturally grown bulk crystals of $\mathrm{Bi}_{2} \mathrm{Se}_{3}$, the measured quantum oscillations in most cases are dominated by bulk electrons $[47,48,52,53]$. To mitigate bulk contribution, one could shift the $E_{F}$ to lower energies or reduce the sample size. The former can be achieved by front/back gating or chemical doping [51,54-56]. The latter can be realized in nanosized TI samples with a much higher surface-to-bulk ratio that can be obtained by exfoliating bulk crystals using the "scotch tape method" or by synthesizing nanowires or nanoribbons.

A widely adopted method to grow $\mathrm{Bi}_{2} \mathrm{Se}_{3}$ and $\mathrm{Bi}_{2} \mathrm{Te}_{3}$ nanowires is the so-called vapor-liquid-solid growth (VLSG). The details of the growth mechanism and experimental setup can be found in references [57-62]. Quantum oscillation measurements on TI nanoribbons were reported in [58,63-65]. The surface state can be identified by angle-dependent magneto-resistance measurements. Due to the 2D characteristic of the topological surface state, the oscillatory magneto-resistance in tilted magnetic fields must satisfy a scaling law $\Delta R(B, \theta) \sim f(B \cos \theta)$, where $\Delta R$ represents the oscillation amplitude, $B$ denotes the magnetic field and $\theta$ is an angle between the tilted field and the $c$-axis of the crystal. An example of the Shubnikov-de Haas quantum oscillation of the surface state of a $\mathrm{Bi}_{2} \mathrm{Se}_{3}$ nanoribbon is delineated in Fig. 2(a). The nanoribbon, shown in the inset of Fig. 2(b), was grown using a catalyst-free physical vapor deposition method [65]. This method can grow millimeter-long nanoribbons with excellent surface quality, underlined by the high mobility of the Dirac electrons. The oscillations measured in tilted fields from 0 to $50^{\circ}$ collapse into one curve when the horizontal coordinate is expressed as $1 /(B \cos \theta)$. This $2 \mathrm{D}$ scaling of the field-dependent quantum oscillation is a strong evidence of the topological surface state. Applying the classical Lifshitz-Kosevich (LK) theory, parameters of the Dirac electrons, such as the effective mass $m^{*}$ and scattering time $\tau$, can be extracted from the oscillation frequency and the temperature-dependent oscillation amplitude. The detailed analysis of quantum oscillation using LK theory can be found in the book "Magnetic Oscillations in Metals" by Shoenberg [66] and in review articles [67-70]. As aforementioned, the helical spin structure of TIs generates a $\pi$-Berry's phase of the electrons when the spin rotates by 

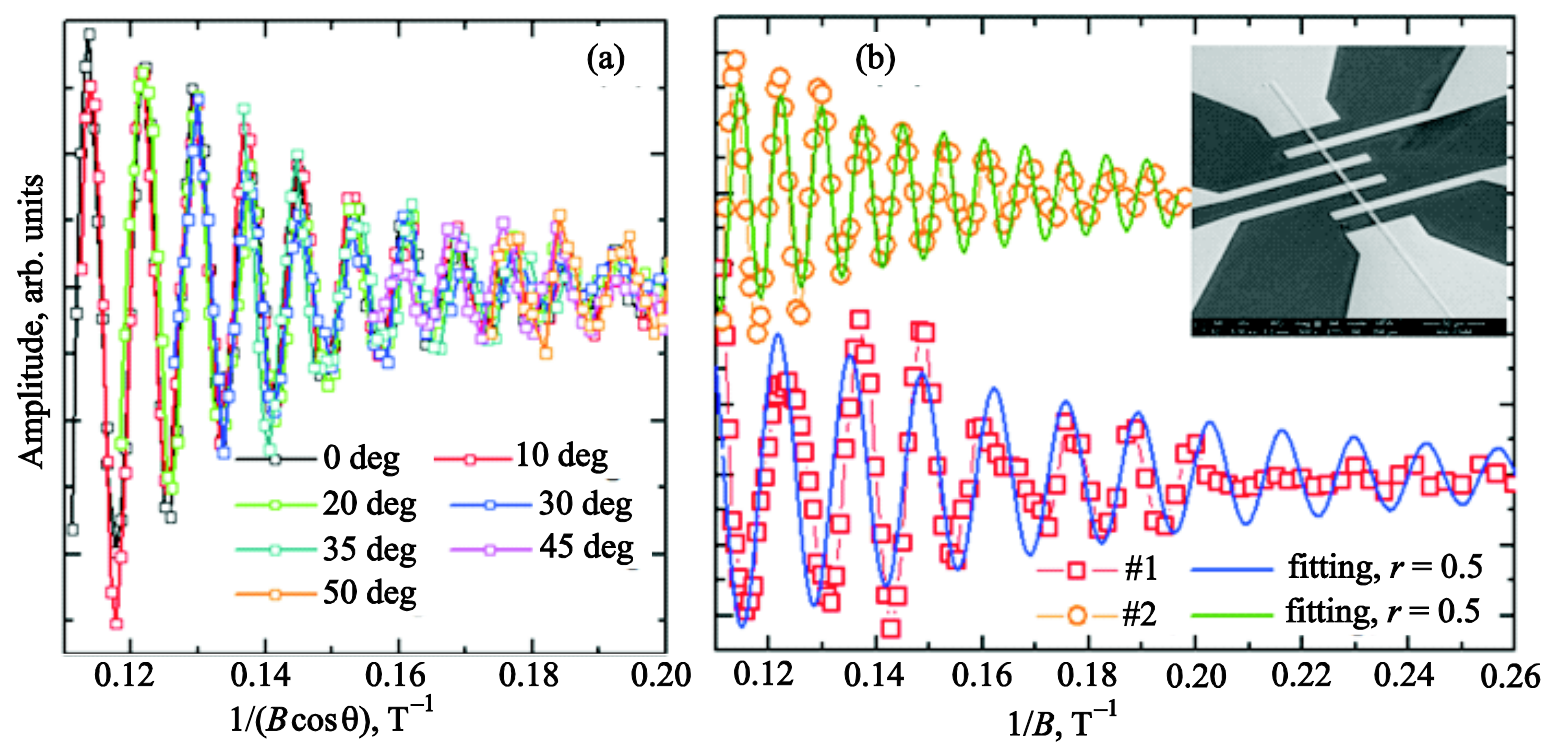

Fig. 2. (Color online) The scaled Shubnikov-de Haas quantum oscillations measured in a $\mathrm{Bi}_{2} \mathrm{Se}_{3}$ nanoribbon. The $2 \mathrm{D}$ characteristic suggests that it originates from the surface state (a). The determination of Berry's phase using the LK theory. A phase factor of $1 / 2$ is consistently observed in different $\mathrm{Bi}_{2} \mathrm{Se}_{3}$ nanoribbon. The inset is a scanning electron microscopy picture of nanoribbon (b).

$2 \pi$ around the basal plane of the Dirac cone. The $\pi$-Berry's phase, the hallmark of the Dirac electron, can be extracted from the field dependent quantum oscillations. In LK theory and in a 2D system, the oscillatory resistance $\Delta R \sim \cos [2 \pi(F / B+1 / 2+r)]$, where the $F$ is the oscillation frequency. The Berry's phase is represented by the phase factor $r$, which is equal to 0 or $1 / 2$ for conventional Schrödinger electrons and Dirac electrons, respectively [71]. As shown in Fig. 2(b), a phase factor of $1 / 2$ is consistently observed in different $\mathrm{Bi}_{2} \mathrm{Se}_{3}$ nanoribbons. The $\pi$-Berry's phase is another evidence of the existence of the surface state. On the other hand, the phase factor $r$ is the same quantity as the " $r$ " in Onsager's quantization condition, $A_{n}=(2 \pi e / \hbar) B(n+r)$, where $A_{n}$ is the Fermi surface crosssection area. The Onsager relation provides another approach to determine the phase factor, i.e., the fan diagram of Landau-levels [71,72]. In this diagram, $r$ can be obtained by linearly extrapolating the $1 / B$-vs- $n$ curve to $1 / B=0$. However, large ambiguities may occur if the linear extrapolation is not carefully performed, especially when there are insufficient data points in the fan diagram [73]. Therefore, a reliable determination of the value of $r$ using this approach requires a certain number of quantum oscillation cycles, in other words, a high-enough magnetic field.

\subsection{Weak antilocalization}

Another quantum phenomenon frequently observed in transport measurements is the weak antilocalization effect. In a disordered system, electron elastic scattering (e.g. scattering with impurities) dominates over inelastic scattering (e.g. scattering with phonons) at low temperatures. Since the phase of the electron is retained after elastic scattering but is lost after inelastic scattering, the phase coherence length, $l_{\varphi}$, of the electrons is much longer than the mean free path, $l_{e}$. Here, the $l_{\varphi}$ is defined as the distance that an electron travels before its phase is lost, and the $l_{e}$ is defined as the distance that an electron travels before it is scattered, in this case, by an elastic scattering center. The phase interference between electrons propagating on intersecting paths increases the probability of electrons to remain at their initial location. This process is referred to as weak localization [74]. The weak localization effect is schematically depicted in Fig. 3(a). The weak localization leads to a negative correction in the total conductivity. At zero field, the temperature dependence of the conductivity correction is expressed as $\Delta \sigma(T, B=0)=-\left(e^{2} / 2 \pi h\right) \ln T$, where the $h$ is the Planck constant. In a system with strong SOC, the spin rotation during the electron propagation gives rise to a destructive interference, which leads to a higher net conductivity. This destructive interference is called weak antilocalization (WAL) [74]. In TIs, the $\pi$-Berry's phase also destroys the interference of electrons and gives rise to the WAL $[46,63,75-79]$.

In a 2D system, the conductivity correction at applied magnetic field from either weak localization or WAL can be described by the Hikami-Larkin-Nagaoka (HLN) equation [80],

$$
\Delta \sigma(B) \cong-\alpha \frac{e^{2}}{\pi \hbar}\left[\psi\left(\frac{1}{2}\right)+\frac{B_{\varphi}}{B}-\ln \left(\frac{B_{\varphi}}{B}\right)\right]
$$

where $\alpha$ is $-1 / 2$ for weak localization and is $1 / 2$ for WAL; $\Psi(z)$ is the digamma function; $B_{\varphi}=\hbar /\left(4 e l_{\varphi}\right)$ is the characteristic field associated with the coherence length $l_{\varphi}$ : $l_{\varphi}=\sqrt{D \tau_{\varphi}}$ and $D$ is the diffusion constant, $D=(1 / 2) v_{F}^{2} \tau_{e}$ in a 2D system, where $v_{F}$ is the Fermi velocity and $\tau_{e}$ is the elastic scattering time; $\hbar$ is Planck constant divided by $2 \pi$. 
(a)

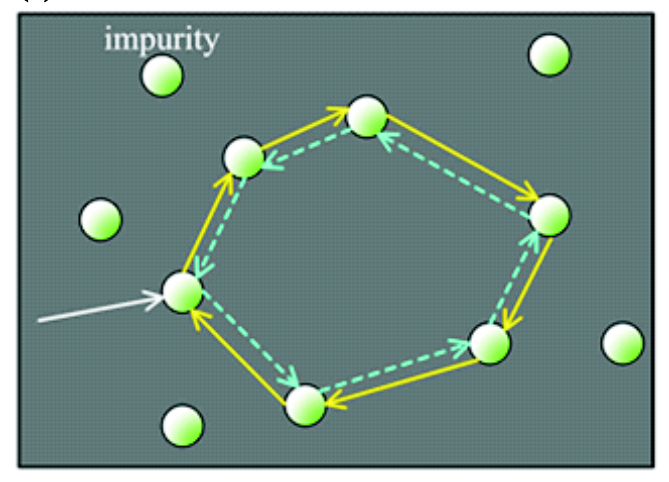

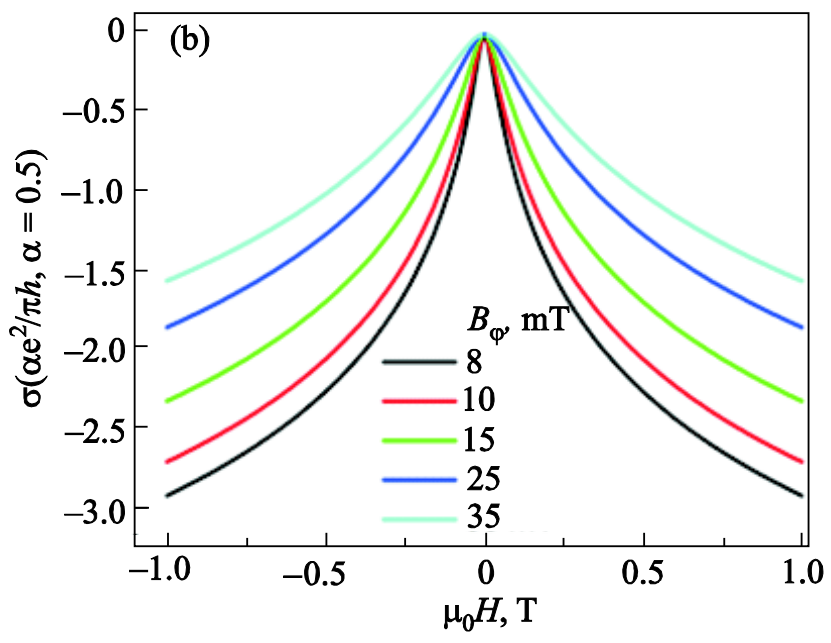

Fig. 3. (Color online) A schematic picture of the weak localization effect. The solid yellow curve and the dashed blue curve represent a pair of time reversed paths, which form a constructive interference to localize the electrons. This interference can be destroyed by either the strong SOC or the Berry's phase of the Dirac electron in TIs (a). Theoretical curves of the corrected conductivity of the weak antilocalization effect. The smaller value of the characteristic field $B_{\varphi}$ corresponds to a longer phase coherence length $l_{\varphi}$ (b).

The phase coherence length of the 2D WAL can be quantitatively obtained by fitting the $\Delta \sigma(B)$. Figure 3(b) shows the theoretical curves of the WAL for different values of $B_{\varphi}$. For $\mathrm{Bi}_{2} \mathrm{Te}_{3}$ the reported value of $l_{\varphi}$ is about $300 \mathrm{~nm}$ $[76,77]$. The WAL was observed in several magnetotransport measurements, and has been associated with the $\pi$-Berry's phase of the 2D Dirac fermions. However, the observation of 2D WAL is not a decisive proof of the existence of Dirac electrons. As a matter of fact, strong SOC, the prerequisite of TIs, can also lead to WAL $[74,81]$. For TI samples with 2D geometry, WAL induced by SOC of bulk electrons and $\pi$-Berry's phase of Dirac electrons are indistinguishable. This issue can be addressed in 3D samples. Magneto-conductivity measurement on thin film $\mathrm{Bi}_{2} \mathrm{Se}_{3}$ with a thickness of $50 \mathrm{~nm}$ has successfully separated the 2D WAL from the 3D bulk contribution [76,77]. Some interesting properties of WAL in TIs were revealed by thickness dependent transport measurements on fewlayer $\mathrm{Bi}_{2} \mathrm{Se}_{3}$ thin films $[78,82]$. Due to the hybridization between the top and bottom surface states, a gap appears at the Dirac point. Under this condition, the WAL was found to coexist with weak localization. The WAL is not limited to $\mathrm{Bi}_{2} \mathrm{Se}_{3}$ and $\mathrm{Bi}_{2} \mathrm{Te}_{3}$. Recently, an observation of the 2D WAL was reported in TI $(\mathrm{PbSe})_{5}\left(\mathrm{Bi}_{2} \mathrm{Se}_{3}\right)_{6}$ [83,84]. WAL was also observed in the strongly correlated topological Kondo insulator $\mathrm{SmB}_{6}[85,86]$.

\subsection{Aharonov-Bohm effect and Altshuler-Aronov-Spivak effect}

In addition to the WAL, phase coherence in TI also gives rise to the Aharonov-Bohm (AB) effect [87] and the Altshuler-Aronov-Spivak (AAS) effect [88]. Cui's group at Stanford University first observed the oscillatory magnetoresistance in $\mathrm{Bi}_{2} \mathrm{Se}_{3}$ nanoribbons at low temperatures [57]. Analysis using fast Fourier transformation revealed a period of $h / e$ in the oscillatory magnetoresistance.
This oscillation was ascribed to the $\mathrm{AB}$ effect in a TI nanoribbon where a surface current circulates around the perimeter of the ribbon. However, given the constructive interference between electrons on pairs of time-reversed paths, the AAS effect with an oscillatory period of $h / 2 e$ is expected. The explanation was that the backscattering immunity, one of the features of TI, destroys the constructive interference [57]. Recently, both $\mathrm{AB}$ and AAS effects were observed in $\mathrm{Bi}_{2} \mathrm{Te}_{3}$ nanoribbons [64]. Although the $h / e$ oscillatory period was observed repeatedly in two TIs, its origin is still not fully understood $[89,90]$. It is worth pointing out that the surface of $\mathrm{Bi}_{2} \mathrm{Se}_{3}$ is not stable. The surface band structure varies with time. ARPES has observed a striking splitting between the spin-up band and spin-down band on the surface of $\mathrm{Bi}_{2} \mathrm{Se}_{3}$ [9]. Consequences such as inter-band scattering are likely to be induced. These effects could complicate the phase coherence in TI nanoribbons and give rise to the anomalous oscillatory period $h / e$. These hypotheses, however, need future investigation. The $\mathrm{AB}$ effect is not limited to nanowires of $\mathrm{Bi}_{2} \mathrm{Se}_{3}$ and $\mathrm{Bi}_{2} \mathrm{Te}_{3}$. In fact, an h/e oscillatory period was recently observed in nanowires of $\beta-\mathrm{Ag}_{2} \mathrm{Te}$ [91]. Angle and temperature dependences of the $\mathrm{AB}$ oscillation demonstrated 2D features of the surface state in this material, consistent with the theoretical prediction of a topological state in $\beta-\mathrm{Ag}_{2} \mathrm{Te}$ [92].

\section{Summary and prospect}

In this article, we briefly reviewed the progresses in the transport measurements of TIs. We discussed the quantum oscillation, weak antilocalization, AB/AAS effect, together with the identification of the topological surface states using these quantum phenomena. Some aspects such as strong electron-electron correlation and spin analogues to 
the fractional quantum Hall effect were not included in this review because they are not yet fully understood. The application of TIs in spin transport devices seems promising as both theory and experiments in optical spectroscopy have indicated the potential of $\mathrm{TI}$ in the application of spintronics [6,93]. Furthermore, noticeable efforts are being devoted to designing novel devices to disentangle the spin-up and spin-down electrons in TIs. Rapid progress in this particular field can be expected. The research in TIs is also expanding into particle physics. A combination of TI nanowires with the superconducting proximity effect and magnetic fields is predicted to host the long-sought Majorana fermion [94], a particle which was hypothesized to be the sole component of dark matter in our universe. Currently, a "Majorana race" is blooming all over the world $[95,96]$. Another discovery of particle physics, following the observation of the Higgs boson, may be achieved in the condensed matter community.

To conclude, in the journey to master topological insulators, numerous novel physics and materials have been discovered. The momentum for further discoveries will not be abated since current theoretical tools are adequate and powerful enough to enable realistic predictions. The progress towards application of topological insulators should come with optimism, given the strong integration of advanced materials synthesis, powerful characterizations, device modeling and fabrications, and theories.

LF and WKK thank the support of the Department of Energy, Office of Basic Energy Sciences, under Contract No. DE-AC02-06CH11357.

1. N.W. Ashcroft and N.D. Mermin, Solid State Physics, Thomson Learning, Inc. (1976).

2. C.C. Tsuei and J.R. Kirtley, Rev. Mod. Phys. 72, 969 (2000).

3. C.L. Kane and E.J. Mele, Phys. Rev. Lett. 95, 226801 (2005).

4. C.L. Kane and E.J. Mele, Phys. Rev. Lett. 95, 146802 (2005).

5. B.A. Bernevig and S.C. Zhang, Phys. Rev. Lett. 96, 106802 (2006).

6. B.A. Bernevig, T.L. Hughes, and S.-C. Zhang, Science 314, 1757 (2006).

7. M. König, S. Wiedmann, C. Brüne, A. Roth, H. Buhmann, L.W. Molenkamp, X.-L. Qi, and S.-C. Zhang, Science 318, 766 (2007).

8. D. Pesin and A.H. MacDonald, Nature Mater. 11, 409 (2012).

9. P.D.C. King, R.C. Hatch, M. Bianchi, R. Ovsyannikov, C. Lupulescu, G. Landolt, B. Slomski, J.H. Dil, D. Guan, J.L. Mi, E.D.L. Rienks, J. Fink, A. Lindblad, S. Svensson, S. Bao, G. Balakrishnan, B.B. Iversen, J. Osterwalder, W. Eberhardt, F. Baumberger, and Ph. Hofmann, Phys. Rev. Lett. 107, 096802 (2011).

10. A.Yu. Kitaev, Ann. Phys. 303, 2 (2003).

11. S. Das Sarma, M. Freedman, and C. Nayak, Phys. Rev. Lett. 94, 166802 (2005).
12. L. Fu and C.L. Kane, Phys. Rev. Lett. 100, 096407 (2008).

13. L. Fu and C.L. Kane, Phys. Rev. B 79, 161408(R) (2009).

14. M.Z. Hasan and C.L. Kane, Rev. Mod. Phys. 82, 3045 (2010).

15. M.Z. Hasan and J.E. Moore, Ann. Rev. Cond. Mat. Phys. 2, 55 (2010).

16. X.-L. Qi and S.-C. Zhang, Rev. Mod. Phys. 83, 1057 (2011).

17. X.-L. Qi, and S.-C. Zhang, Phys. Today 63, 33 (2010).

18. J.E. Moore, Nature 464, 194 (2010).

19. M. König, H. Buhmann, L.W. Molenkamp, T. Hughes, C.X. Liu, X.L. Qi, and S.C. Zhang, J. Phys. Soc. Jpn. 77, 031007 (2008).

20. R.J. Cava, H.W. Ji, M.K. Fuccillo, Q.D. Gibson, and Y.S. Hor, J. Mater. Chem. C 1, 3176 (2013).

21. Y. Ando, J. Phys. Soc. Jpn. 82, 102001 (2013).

22. J.E. Moore and L. Balents, Phys. Rev. B 75, 121306 (2007).

23. L. Fu, C.L. Kane, and E.J. Mele, Phys. Rev. Lett. 98, 106803 (2007).

24. L. Fu and C.L. Kane, Phys. Rev. B 76, 045302 (2007).

25. H.J. Zhang, C.-X. Liu, X.-L. Qi, X. Dai, Z. Fang, and S.-C. Zhang, Nat. Phys. 5, 438 (2009).

26. D. Hsieh, D. Qian, L. Wray, Y. Xia, Y.S. Hor, R.J. Cava, and M.Z. Hasan, Nature 452, 970 (2008).

27. D. Hsieh, Y. Xia, D. Qian, L. Wray, J.H. Dil, F. Meier, J. Osterwalder, L. Patthey, G.J. Checkelsky, N.P. Ong, A.V. Fedorov, H. Lin, A. Bansil, D. Grauer, Y.S. Hor, R.J. Cava, and M.Z. Hasan, Nature 460, 1101 (2009).

28. D.-X. Qu, Y.S. Hor, J. Xiong, R.J. Cava, and N.P. Ong, Science 329, 821 (2010).

29. J.G. Analytis, R.D. McDonald, S.C. Riggs, J.-H., Chu, G.S. Boebinger, and I.R. Fisher, Nat. Phys. 6, 960 (2010).

30. A.A. Taskin and Y. Ando, Phys. Rev. B 80, 085303 (2009).

31. Y.L. Chen, J.G. Analytis, J.-H. Chu, Z.K. Liu, S.-K. Mo, X.L. Qi, H.J. Zhang, D.H. Lu, X. Dai, Z. Fang, S.C. Zhang, I.R. Fisher, Z. Hussain, and Z.-X. Shen, Science 325, 178 (2009).

32. D. Hsieh, Y. Xia, L. Wray, D. Qian, A. Pal, J.H. Dil, J. Osterwalder, F. Meier, G. Bihlmayer, C.L. Kane, Y.S. Hor, R.J. Cava, and M.Z. Hasan, Science 323, 919 (2009)

33. R. Yu, W. Zhang, H.-J. Zhang, S.-C. Zhang, X. Dai, and Z. Fang, Science 329, 61 (2010).

34. C.-Z. Chang, J. Zhang, X. Feng, J. Shen, Z. Zhang, M. Guo, K. Li, Y. Ou, P. Wei, L.-L. Wang, Z.-Q. Ji, Y. Feng, S. Ji, Xi Chen, J. Jia, X. Dai, Z. Fang, S.-C. Zhang, K. He, Y. Wang, L. Lu, X.-C. Ma, and Q.-K. Xue, Science 340, 167 (2013).

35. J.G. Checkelsky. J. Ye, Y. Onose, Y. Iwasa and Y. Tokura, Nat. Phys. 8, 729 (2012).

36. K. von Klitzing, G. Dorda, and M. Pepper, Phys. Rev. Lett. 45, 494 (1980).

37. D.J. Thouless, M. Kohmoto, M.P. Nightingale, and M. den Nijs, Phys. Rev. Lett. 49, 405 (1982).

38. B.I. Halperin, Phys. Rev. B 25, 2185 (1982).

39. S. Murakami, N. Nagaosa, and S.-C. Zhang, Phys. Rev. Lett. 93, 156804 (2004).

40. F.D.M. Haldane, Phys. Rev. Lett. 61, 2015 (1988).

41. H.A. Kramers, Proc. Amsterdam Acad. 33, 959 (1930). 
42. D. Hsieh, Y. Xia, D. Qian, L. Wray, F. Meier, J.H. Dil, J. Osterwalder, L. Patthey, A.V. Fedorov, H. Lin, A. Bansil, D. Grauer, Y.S. Hor, R.J. Cava, and M.Z. Hasan, Phys. Rev. Lett. 103, 146401 (2009).

43. P. Roushan, J. Seo, C.V. Parker, Y.S. Hor, D. Hsieh, D. Qian, A. Richardella, M.Z. Hasan, R.J. Cava, and Ali Yazdani, Nature 460, 1106 (2009).

44. T. Zhang, P. Cheng, X. Chen, J.-F. Jia, X.C. Ma, K. He, L. Wang, H. Zhang, X. Dai, Z. Fang, X.C. Xie, and Qi-Kun Xue, Phys. Rev. Lett. 103, 266803 (2009).

45. Z. Alpichshev, J.G. Analytis, J.-H. Chu, I.R. Fisher, Y.L. Chen, Z.X. Shen, A. Fang, and A. Kapitulnik, Phys. Rev. Lett. 104, 016401 (2010).

46. J. Chen, H.J. Qin, F. Yang, J. Liu, T. Guan, F.M. Qu, G.H. Zhang, J.R. Shi, X.C. Xie, C.L. Yang, K.H. Wu, Y.Q. Li, and L. Lu, Phys. Rev. Lett. 105, 176602 (2010).

47. J.G. Analytis, J.-H. Chu, Y.L. Chen, F. Corredor, R.D. McDonald, Z.X. Shen, and I.R. Fisher, Phys. Rev. B 81, 205407 (2010).

48. K. Eto, Z. Ren, A.A. Taskin, K. Segawa, and Y. Ando, Phys. Rev. B 81, 195309 (2010).

49. A.A. Taskin, Z. Ren, S. Sasaki, K. Segawa, and Y. Ando, Phys. Rev. Lett. 107, 016801 (2011).

50. J. Xiong, A.C. Petersen, D.X. Qu, R.J. Cava, and N.P. Ong, Physica E 44, 917 (2012).

51. Z. Ren, A.A. Taskin, S. Sasaki, K. Segawa, and Y. Ando, Phys. Rev. B 82, 241306(R) (2010).

52. N.P. Butch, K. Kirshenbaum, P. Syers, A.B. Sushkov, G.S. Jenkins, H.D. Drew, and J. Paglione, Phys. Rev. B 81, 241301 (2010).

53. H. Cao, J. Tian, I. Miotkowski, T. Shen, J. Hu, S. Qiao, and Y.P. Chen, Phys. Rev. Lett. 108, 216803 (2012).

54. S. Jia, H. Ji, E. Climent-Pascual, M.K. Fuccillo, M.E. Charles, J. Xiong, N.P. Ong, and R.J. Cava, Phys. Rev. B 84, 235206 (2011).

55. D.S. Kong, Y.L. Chen, J.J. Cha, Q.F. Zhang, J.G. Analytis, K. Lai, Z.K. Liu, S.S. Hong, K.J. Koski, S.-Kwan Mo, Z. Hussain, I.R. Fisher, Z.-X. Shen, and Y. Cui, Nature Nanotechnology 6, 705 (2011).

56. H. Steinberg, D.R. Gardner, Y.S. Lee, and P. Jarillo-Herrero, Nano Lett. 10, 5032 (2010).

57. H.L. Peng, K. Lai, D. Kong, S. Meister, Y.L. Chen, X.-L. Qi, S.-C. Zhang, Z.-X. Shen, and Y. Cui, Nature Mater. 9, 225 (2010).

58. H. Tang, D. Liang, R.L.J. Qiu, and Xuan P.A. Gao, ACS Nano 5, 7510 (2011).

59. S.S. Hong, W. Kundhikanjana, J.J. Cha, K. Lai, D. Kong, S. Meister, M.A. Kelly, Z.-X. Shen, and Yi Cui, Nano Lett. 10, 3118 (2010).

60. D.S. Kong, J.C. Randel, H. Peng, J.J. Cha, S. Meister, K. Lai, Y.L. Chen, Z.-X. Shen, H.C. Manoharan, and Y. Cui, Nano Lett. 10, 329 (2010).

61. Jonathan E. Allen, Eric R. Hemesath, Daniel E. Perea, Jessica L. Lensch-Falk, Z.Y. Li, Feng Yin, Mhairi H. Gass, Peng Wang, Andrew L. Bleloch, Richard E. Palmer, and Lincoln J. Lauhon, Nat. Nanotechnol. 3, 168 ( 2008).
62. Y. Xia, P. Yang, Y. Sun, Y. Wu, B. Mayers, B. Gates, Y. Yin, F. Kim, and H. Yan, Adv. Mater. 15, 353 (2003).

63. S.S. Hong, J.J. Cha, D.S. Kong, and Yi Cui, Nature Commun. 3, 757 (2012).

64. F.X. Xiu, L. He, Y. Wang, L. Cheng, L.-T. Chang, M. Lang, G. Huang, X.F. Kou, Y. Zhou, X.W. Jiang, Z.G. Chen, J. Zou, A. Shailos, and K.L. Wang, Nat. Nanotechnol. 6, 216 (2011).

65. L. Fang, Y. Jia, D.J. Miller, M.L. Latimer, Z.L. Xiao, U. Welp, G.W. Crabtree, and W.-K. Kwok, Nano Lett. 12, 6164 (2012).

66. D. Shoenberg, Magnetic Oscillations in Metals, Cambridge University Press, Cambridge, UK (1984).

67. A. Carrington, Rep. Prog. Phys. 74, 124507 (2011).

68. A.I. Coldea, D. Braithwaite, and A. Carrington, Comptes Rendus Phys. 14, 94 (2013).

69. Mark V. Kartsovnik, Chem. Rev. 104, 5737 (2004).

70. Akira Isihara and Ludvig Smrčka, J. Phys. C: Solid State Phys. 19, 6777 (1986).

71. G.P. Mikitik and Yu.V. Sharlai, Phys. Rev. Lett. 82, 2147 (1999).

72. Y.B. Zhang, Y.-W. Tan, H.L. Stormer, and P. Kim, Nature 438, 201 (2005).

73. A.A. Taskin and Yoichi Ando, Phys. Rev. B 84, 035301 (2011).

74. V.F. Gantmakher, Electrons and Disorder in Solids, Clarendon Press, Oxford (2005).

75. J. Chen, X.Y. He, K.H. Wu, Z.Q. Ji, L. Lu, J.R. Shi, J.H. Smet, and Y.Q. Li, Phys. Rev. B 83, 241304 (2011).

76. Hong-Tao He, Gan Wang, Tao Zhang, Iam-Keong Sou, George K.L. Wong, and Jian-Nong Wang, Phys. Rev. Lett. 106, 166805 (2011).

77. L. Bao, L. He, N. Meyer, X. Kou, P. Zhang, Z.-G Chen, A.V. Fedorov, J. Zou, T.M. Riedemann, T.A. Lograsso, K.L. Wang, G. Tuttle, and F.X. Xiu, Scient. Rep. 2, 726 (2012).

78. M. Lang, L. He, X. Kou, P. Upadhyaya, Y. Fan, H. Chu, Y. Jiang, J.H. Bardarson, W. Jiang, E. Sang Choi, Y. Wang, N.-C. Yeh, J. Moore, and K.L. Wang, Nano Lett. 13, 48 (2013).

79. M. Liu, J. Zhang, C.-Z. Chang, Z.C. Zhang, X. Feng, K. Li, K. He, L.-L. Wang, X. Chen, X. Dai, Z. Fang, Q.-K. Xue, X.C. Ma, and Y.Y. Wang, Phys. Rev. Lett. 108, 036805 (2012).

80. S. Hikami, A.I. Larkin, and Y. Nagaoka, Prog. Theor. Phys. 63, 707 (1980).

81. G. Bergmann, Phys. Rep. 107, 1 (1984).

82. A.A. Taskin, S. Sasaki, K. Segawa, and Y. Ando, Phys. Rev. Lett. 109, 066803 (2012).

83. L. Fang, C.C. Stoumpos, Y. Jia, A. Glatz, D.Y. Chung, H. Claus, U. Welp, W.K. Kwok, and M.G. Kanatzidis, arXiv:1307.0260.

84. K. Nakayama, K. Eto, Y. Tanaka, T. Sato, S. Souma, T. Takahashi, Kouji Segawa, and Yoichi Ando, Phys. Rev. Lett. 109, 236804 (2012).

85. S. Thomas, D.J. Kim, S.B. Chung, T. Grant, Z. Fisk, and Jing Xia, arXiv:1307.4133. 
86. M. Dzero, K. Sun, V. Galitski, and P. Coleman, Phys. Rev. Lett. 104, 106408 (2010).

87. Y. Aharonov and D. Bohm, Phys. Rev. 115, 485 (1959).

88. B.L. Altshuler, A.G. Aronov, and B.Z. Spivak, JETP Lett. 33, 94 (1981).

89. J.H. Bardarson, P.W. Brouwer, and J.E. Moore, Phys. Rev. Lett. 105, 156803 (2010).

90. Y. Zhang and A. Vishwanath, Phys. Rev. Lett. 105, 206601 (2010).

91. S. Lee, J. In, Y. Yoo, Y. Jo, Y.C. Park, H.-J. Kim, H.C. Koo, J. Kim, B. Kim, and K.L. Wang, Nano Lett. 12, 4194 (2012).
92. W. Zhang, R.Yu, W. Feng, Y. Yao, H. Weng, X. Dai, and Z. Fang, Phys. Rev. Lett. 106, 156808 (2011).

93. A.A. Burkov and D.G. Hawthorn, Phys. Rev. Lett. 105, 066802 (2010).

94. For a review, Jason Alicea, Rep. Prog. Phys. 75, 076501 (2012) and references therein.

95. M. Franz, Physics 3, 24 (2010).

96. M. Franz, Nat. Nanotechnol. 8, 149 (2013). 